\title{
LA INVESTIGACIÓN SOBRE LOS ESTILOS DE ENSEÑANZA. APORTES PARA MEJORAR LA DIDÁCTICA DE CIENCIAS
}

\author{
RESEARCH ABOUT TEACHING STYLES. \\ CONTRIBUTIONS TO IMPROVE TEACHING SCIENCE
}

\author{
Ana Clara Ventura* \\ Consejo Nacional de Investigaciones Científicas y Técnicas - CONYCET, Argentina
}

\section{RESUMEN}

El objetivo del presente artículo es precisar aspectos conceptuales y operacionales del constructo "estilos de enseñanza" marcando sus tres propiedades distintivas: relativa estabilidad, multidimensionalidad y neutralidad valorativa. Asimismo, se analizan distintas investigaciones empíricas sobre el tema sintetizando algunos de los puntos más compartidos: los docentes transmiten conocimientos así como formas típicas de aprendizaje; los estilos de enseñanza se diferencian de acuerdo con el tipo de disciplina enseñada; el ajuste entre los estilos de enseñanza de los docentes y los estilos de aprendizaje de los estudiantes facilita la adquisición de conocimientos. En síntesis, este trabajo pretende aportar conocimientos y estrategias que contribuyan a la formación en didáctica de ciencias y, por ende, al fortalecimiento de prácticas eficaces de enseñanza.

Palabras clave: enseñanza de ciencias, estrategias educativas, educación superior.

\begin{abstract}
The aim of this paper is to clarify conceptual and operational aspects of the construct "teaching styles" with its three distinctive properties: relative stability, multidimensionality and value neutrality. Moreover, it analyzes the points of view that are shared for many empirical studies: teachers transmit knowledge and typical forms of learning; teaching styles differ according to the type of subject taught, the match between the teaching styles and learning styles improve knowledge acquisition. In summary, this paper provides knowledge and strategies that contribute to training in science teaching and, therefore, to strengthen effective teaching practices.
\end{abstract}

Keywords: teaching science, educational strategies, higher education. 


\section{INTRODUCCIÓN}

Desde una perspectiva didáctica de la enseñanza de ciencias, se reconoce que la construcción de los conocimientos en el aula universitaria se configura mediante la interacción entre docentes, los alumnos y los contenidos. Por su parte, Gagliardi (2008) integró los componentes didácticos centrales en un modelo pedagógico orientado hacia la formación de estudiantes autónomos: la determinación de conceptos estructurantes, la exploración de concepciones y obstáculos al aprendizaje, la planificación de la organización temporalasí como dela orientación vocacional y, por último, la identificación de los estilos de aprendizaje así como de los estilos de enseñanza.

Con base en una revisión bibliográfica, se observó que estos aspectos didácticos fueron investigados en distintos escenarios educativos y contextos geográficos (Aguilera, 2012; Benito, 2009; De la Fuente Arias, Salvador Granados \& De la Fuente Arias, 1992; Duit, 2006; Ortiz \& Mariño, 2007; Petrucci \& Dibar, 2001; Pozo, Sanz, Gómez \& Limón, 1991, Viale, 2007, 2011). No obstante, es posible apreciar que preexiste cierta vacancia teórica y metodológica acerca de los estilos de enseñanza (Breijo, 2005; De León, 2005; Martínez, 2009).

Guzmán (2009) explica que es común asumir dos concepciones acerca de la enseñanza en el ámbito universitario. Por un lado, los profesores no requieren una formación pedagógica especial para enseñar dado que el dominio de la disciplina los habilita automáticamente para enseñarla; por otro lado, la docencia como una actividad sencilla que cualquiera puede desempeñar con relativa facilidad. Debido a ello, se considera que la innovación de la docencia universitaria es menor respecto a otros ciclos educativos.

En línea con estas ideas, Camargo \& Hederich
(2007) especifican que las investigaciones y debates focalizados en los estilos de enseñanza parecen estar en sus fases iniciales dado que son pocos los modelos teóricos explicativos que se han construido y presentan escaso respaldo científico en sus formulaciones.

Por lo expuesto, el propósito de este artículo es delimitar aspectos conceptuales y operacionales del constructo "estilos de enseñanza". Asimismo, se analizan distintas investigaciones empíricas sobre el tema y se destacan los puntos en donde se sitúan los mayores grados de acuerdo científico: 1. La trasmisión de conocimientos así como formas típicas de aprendizaje por parte de los docentes; 2. La diferenciación de los estilos de enseñanza de acuerdo con el tipo de disciplina enseñada, $y$, 3. El emparejamiento entre estilos de enseñanza y aprendizaje facilita el desarrollo conceptual y desempeño de los estudiantes.

Cabe señalar que la principal intención del artículo consiste en efectuar una caracterización resumida acerca de los enfoques y postulados compartidos en la actualidad con el fin de identificar núcleos problemáticos que orienten el trabajo de futuras investigaciones. A nivel de sus implicancias educativas, se pretende brindar herramientas conceptuales y prácticas que contribuyan a la formación docente en didáctica de ciencias y, por ende, al fortalecimiento de prácticas efectivas de enseñanza.

\section{Hacia una conceptualización de los estilos de enseñanza}

Los estilos de enseñanza aluden a la forma personal que tiene cada docente de pensar y actuar dentro del entorno de aula (Pinelo, 2008). "La literatura sobre estilos de docencia o enseñanza es abundante y muestra diferentes 
clasificaciones (...) sin embargo, todos los estudios que abordan este tema hacen referencia a un conjunto de habilidades y prácticas propias de la docencia que caracterizan formas de enseñanza relativamente estables" (Borgobello, Peralta y Roselli, 2010, p.9) configuradas con base en rasgos e historias personales así como a teorías implícitas de la enseñanza y el aprendizaje (Roselli, 2011).

En este sentido, un primer acuerdo conceptualsupondría quelos estilos deenseñanza no remiten a qué se enseña, sino a cómo los docentes prefieren enseñar y cuáles son sus procedimientos típicos o predominantemente empleados en el acto de enseñar.

Por otro lado, es frecuente encontrar que las expresiones "estrategias", "métodos" y "estilos" de enseñanza se utilizan en forma equivalente. Sin embargo, es preciso señalar ciertas divergencias entre los términos. Las estrategias son herramientas utilizadas por un sujeto para facilitar la adquisición de conocimientos; se caracterizan por ser intencionales y producto del entrenamiento (Beltrán, Alcillo, Pérez \& Rodríguez, 2005). En íntima vinculación con las estrategias, se ubican los métodos que remiten a los planes o maneras de conducir el pensamiento y las acciones para la consecución de un objetivo o finalidad. Un método puede incluir diversas estrategias y técnicas (Beltrán, 1998; Rosales, 2004).

Valadez (2009) distinguió que las estrategias y los métodos son actividades conscientes mientras que los estilos pueden no ser reconocidos por la propia persona, debido a que son configuraciones de rasgos que resultan de disposiciones biológicas y de aprendizaje experiencial (implícito y explícito). A esto se añade que las estrategias y los métodos pueden ser entendidos como el producto de una operación mental y los estilos son procesos fundamentalmente cognitivos y de pensamiento que caracteriza una forma estable de comportarse y de relacionarse en el contexto.

A diferencia de ello, los estilos de enseñanza guardarían mayores grados de asociación conceptual con la expresión "preferencias instruccionales" para enseñar o aprender entendidas como la tendencia de un individuo a ponderar un método instruccional concreto o una combinación de ellos (Clariana, 2001; Tárraga Mínguez, Martínez Monfort, Gómez del Amo, Pastor Cerezuela \& Fernández Andrés).

Una segunda conceptualización indicaría que los estilos de enseñanza remiten al proceso de pensamiento y acción típica en que los docentes se sirven de estrategias y técnicas (productos) de instrucción para enseñar (Figueroa \& Vigliecca, 2006, Laudadio, 2012, Suárez \& Elias, 2008). En este sentido, cuando un docente emplea la secuencia didáctica "lluvia de ideas previas-exposición magistralanálisis de casos" de manera habitual pueden observarse tres estrategias que configuran un estilo de enseñanza típico.

2.1 Propiedades distintivas: estabilidad, multidimensionalidad y neutralidad valorativa

Según Camargo \& Hederich (2007), el concepto general de estilo lleva implícitas las siguientes características:

2.1.1 Es estable, dado que establece diferencias relativamente consistentes en cada individuo. Esta condición posibilita el diagnóstico y medición de los estilos de enseñanza.

2.1.2 Es integradora de diferentes dimensiones del sujeto. Se comparte la idea de que en el constructo de estilos de enseñanza confluyen distintos aspectos o variables. En este marco Roselli (2011) 
sintetizó que cada modelo pone el acento en un aspecto determinado:

"la distribución del poder y de la influencia (concentrados en el docente o distribuidos entre todos los participantes del sistema), la actividad productora del conocimiento (clase expositiva o clase participativa con andamiaje docente), el método de trabajo (individual o por grupos), la intensidad y direccionalidad del intercambio docentealumnos y alumno-alumno (baja o alta interactividad), las características cualitativas de la interacción (incluyendo la actitud física: gestos, mirada, tono de voz y desplazamiento físico) y el involucramiento afectivo y el clima de trabajo (ambiente neutro o afectivamente cálido). Incluso algunas tipologías con explícitamente multidimensiones como la distinción entre estilo tradicional y progresista” (pp.34-35).

Es neutral desde el punto de vista valorativo aludiendo a la imposibilidad de ponderar un estilo por encima de otro en términos absolutos. Numerosas investigaciones quebrantan esta cualidad asumiendo que existen estilos de enseñanza que favorecen por sí mismos el aprendizaje significativo de los estudiantes: e.g. el estilo emancipatorio (Callejas \& Vitalia, 2002), el estilo humanista (Breijo, 2005), el estilo mediacional (Rendón, 2010b), entre otros.

\section{Definición operacional y principales clasificaciones}

Con base en una revisión bibliográfica efectuada, es posible advertir que existe acuerdo en operar el constructo de los estilos de enseñanza al menos en dos niveles de estudio: un nivel general que remite a cuestiones educativas de orden filosófico, epistemológico e institucional en torno a la manera en que se accede al conocimiento, el tipo de comunicaciones y regulaciones entre docente-estudiantes y el control sobre el proceso de aprendizaje. El segundo nivel metodológico-didáctico o nivel específico alude a la materialización de las acciones docentes en el aula (Abello, Hernández \& Hederich, 2011; Caballero, 2007). A continuación, se establecen diferentes tipologías de los estilos de enseñanza.

En este apartado se seleccionaron los modelos de estilos de enseñanza de mayor impacto en las investigaciones latinoamericanas. Por esta razón no resulta una descripción exhaustiva de todas las clasificaciones existentes acerca de los estilos de enseñanza.

Una propuesta clásica y ampliamente aplicada en investigaciones psicoeducativas es la división entre estilos centrados en la enseñanza (con un modelo reproductivo centrado en el profesor y basado en transmisión de información) y estilos centrados en el aprendizaje (con un modelo constructivista centrado en el alumno centrado y enfocado hacia la facilitación del aprendizaje) (Finson, Thomas \& Pedersen, 2006; Gargallo, 2008).

Por otro lado, se sitúa la tipología de Delgado (1991) de orientación eminentemente pedagógica que distingue entre el estilo tradicional (basado en el mando directo y la asignación de tareas), individualizador (centrado en la programación de módulos adaptados a las características del alumnado), participativo (orientado hacia la enseñanza recíproca con pequeños grupos), socializador (centrado en el trabajo colaborativo mediado por juegos de roles y simulaciones), cognoscitivo (basado en el descubrimiento guiado y la resolución de problema) y creativo (tiende hacia el trabajo espontáneo, original incluyendo la sinéctica corporal). 
Por último, se encuentra el modelo de estilos de enseñanza de base fundamentalmente psicológica elaborado por Felder \& Silverman (1988) que distingue entre los estilos concretoabstracto, activo-reflexivo (según el tipo de participación que promueve el docente del alumnado), visual-verbal (de acuerdo con el tipo de soporte que emplea para trasmitir el contenido) y secuencial-global (según el tipo de perspectiva que utiliza para explicar).

\section{Postulados compartidos por investigaciones científicas actuales} las

El profesorado universitario concibe la enseñanza de diferentes formas que se corresponden con connotaciones e implicaciones sobre cómo se espera que los estudiantes aprendan (Ramsden, 1993). Las investigaciones que se centran en el estudio de los estilos de enseñanza asumen que las prácticas educativas involucran no sólo la trasmisión de conceptos sino también de formas de pensar y actuar (Hervás, 2003).

Los enfoques de la enseñanza centrados en el profesor como transmisor de información comienzan a focalizarse en los estudiantes y en las formas de facilitar el proceso de aprendizaje (Feixás, 2010). De aquí que el acto de enseñar es equiparado con la acción de mostrar a los estudiantes las formas de hacer, de pensar, de aprender (Gené, 1998, extraído de Rendón Uribe, 2010a).

Según Irigoyen, Jiménez \& Acuña (2004), el docente, en interacción con el alumno, especifica quehaceres en términos de las operaciones; a partir de estas, el aprendiz observa, manipula, describe y analiza sus resultados bajo los criterios especificados por la disciplina. Es a través de este medio (discurso didáctico) que el docente modela, moldea y demarca criterios de respuesta en la interacción didáctica. Esto es lo que Coll \& Onrubia (1993) han denominado establecimiento de referencias que se construyen progresivamente en el transcurso de las secuencias didácticas.

Por lo expuesto, el primer punto de acuerdo consiste en que los docentes transmiten conocimientos así como formas típicas de aprendizaje mediante sus estilos de enseñanza.

Por otro lado, la mayor parte de estas investigaciones establecen relaciones entre la perspectiva psicológica que se interesa por el aprendizaje y la enseñanza de contenidos y las didácticas específicas que se ocupan de la enseñanza, y el aprendizaje de esos mismos contenidos.

Polanco (1999) demostró que las relaciones entre desempeño docente y su estilo de aprender no son homogéneas, sino que parecen estar claramente matizadas por otros factores, tales como el área de enseñanza. Apoyando esta evidencia, Irigoyen, Jiménez \& Acuña (2003) así como Rendón (2010b) encontraron diferencias en los estilos de enseñanza dependiendo de la disciplina científica estudiada.

Ilustrando estas divergencias, Guzmán (2011) afirmó que los maestros de Ciencias Naturales y Exactas le dan gran importancia al aprendizaje del dominio factual de los hechos y principios de sus disciplinas; en cambio, los de Humanidades y Ciencias sociales otorgan mayor peso al desarrollo personal del estudiante, a la discusión y a las habilidades comunicativas y sociales.

De aquí que la metodología básica de enseñanza, por un lado, en Ciencias Naturales consiste en presentar conceptos claves y ejercicios seguidos de prácticas de laboratorio (Mosquera y Furió-Más, 2008) empleando representaciones gráficas como 
un soporte didáctico y científico elemental (García García \& Perales Palacios, 2005). Por otro lado, en Ciencias Sociales se promueve la integración de estrategias basadas en la cooperación, la interacción y la participación dado que facilitan la construcción de conocimientos complejos (Compagnucci, Cardós \& Ojeda, 2002; Quinquer, 2004).

En este sentido, Pibernat Riera (2010) sintetiza que según la estructura de cada saber será necesario, en el acto de enseñanza, aplicar un tipo de didáctica específica. Por lo expuesto, el segundo acuerdo residiría en que los estilos de enseñanza se diferencian de acuerdo con el tipo de episteme o disciplina enseñada.

Por último, se acuerda que el ajuste/ compatibilidad entre los estilos de enseñanza de los docentes y los estilos de aprendizaje de los estudiantes facilita la adquisición de conocimientos (Aguilera Pupo, 2012; Muros Molina, Som Castillo, Leyva Rodríguez \& Zabala Díaz, 2010; Pastor Martínez, 2010; Pulido Martos, De la Torre-Cruz, Luque Ramos \& Palomo Monereo, 2009; Ramírez Díaz \& Chávez Lima, 2010, Salvador, Argos González, Ezquerra Muñoz, Osoro Sierra y Castro, 2011; Ventura, 2011).

Según Ayala, Díaz \& Orozco (2009) los estudiantes de Fisioterapia de primer año que conocían sus estilos de aprendizaje y en cuya enseñanza se emplearon mapas conceptuales y problemas formulados mediante secuencias neurofuncionales basadas en situaciones de la vida cotidiana, obtuvieron mejores niveles de rendimiento académico que los grupos de años previos.

En un estudio cuasi-experimental, Durán \& Costaguta (2008) compararon el desempeño académico de dos cohortes de estudiantes de Informática. La enseñanza del grupo experimental se ajustó a los estilos de aprendizaje predominantes de los estudiantes (sensitivo-visual-activo). La enseñanza del grupo control no sufrió modificaciones. La intervención generó un mejor rendimiento de los estudiantes en (1) la comprensión de conceptos y métodos, (2) la adquisición de habilidades para la resolución de problemas y (3) el trabajo colaborativo.

Desde el punto de vista de sus implicancias educativas, el encuentro entre los estilos de enseñanza y los estilos de aprendizaje podría establecerse mediante:

- El análisis de los estilos de aprendizaje típicos que exige la disciplina enseñada para que sea socializado y trabajado con los estudiantes. Esta propuesta debería involucrar a todos los actores institucionales, especialmente a los docentes expertos en la disciplina y los especialistas en Educación de la comunidad educativa.

- El fortalecimiento de los estilos de aprendizaje adecuados en los estudiantes mediante sistemas de tutorías o apoyos psicopedagógicos,

- El desarrollo de la capacidad metacognitiva destinada a reconocer críticamente la necesidad de determinados estilos según las exigencias de cada tarea o actividad.

- La diversificación de los recursos, métodos y estilos de enseñanza para generar ambientes educativos óptimos.

\section{CONCLUSIONES}

Los estilos de enseñanza se sitúan como uno de los componentes centrales del proceso educativo desde perspectivas constructivistas de adquisición de conocimientos en el aula universitaria.

Los estilos de enseñanza se caracterizan 
como las formas relativamente estables de los docentes para programar, diseñar y trasmitir conocimientos. Se consideran como aquellos procedimientos preferenciales que se utilizan con base fundamentalmente en teorías implícitas de los docentes acerca de la enseñanza y el aprendizaje, sus necesidades psicológicas, los modos de inicio en la profesión y la cultura académica de la institución.

De aquí la importancia de artículos de este tipo que se ocupan de sistematizar aspectos teóricos y metodológicos dado que se trata de un campo de investigación en vías de consolidación.

En futuras investigaciones empíricas resultaría relevante profundizar sobre:

- La relación entre el estilo de enseñanza y el campo específico del conocimiento o el nivel en la carrera (básico o profesional) - La relación entre los estilos de enseñanza y el pensamiento didáctico de los docentes

- Los modelos de estilos de enseñanza estableciendo acuerdos entre las diferentes taxonomías que existen en la actualidad - La construcción y evaluación de materiales didácticos de formación sobre estilos de enseñanza para docentes $y$ estudiantes universitarios

\section{REFERENCIAS}

Abello, D., Hernández, C., \& Hederich, C. (2011). Estilos de enseñanza en docentes universitarios, propuesta y validación de un modelo teórico e instrumental. Pedagogía y Saberes, 34, 141-154.

Aguilera Pupo, E. (2012). Los estilos de enseñanza, una necesidad para la atención de los estilos de aprendizaje en la educación universitaria. Revista Estilos de Aprendizaje, 10, 79-87.

Aguilera, E. (2012). Los estilos de enseñanza, una necesidad para la atención de los estilos de aprendizaje en la educación universitaria. Revista Estilos de Aprendizaje, 10, 79-87.

Ayala, J., Díaz, J., \& Orozco, J. (2009). Eficacia de la utilización de estilos de aprendizaje en conjunto con mapas conceptuales y aprendizaje basado en la resolución de problemas para el aprendizaje de neuroanatomía. Educación Médica, 12(1), 25-31.

Beltrán, J. (1998). Procesos, estrategias y técnicas de aprendizaje. Madrid, España: Síntesis.

Beltrán, J., Alcillo, I., Pérez, L., \& Rodríguez, E. (2005). Aprender a aprender. Intervención estratégica en estudiantes de secundaria. Revista de Psicología y Educación, 1(2), 35-50.

Benito, M. (2009). Debates en torno a la enseñanza de las ciencias. Perfiles Educativos, 31(123), 27-43.

Borgobello, A., Peralta, N., \& Roselli, N. (2010). El estilo docente universitario en relación al tipo de clase y a la disciplina enseñada. Liberabit, Revista de Psicología, 16(1), 7-16.

Breijo, T. (2005). Los estilos de enseñanza de la historia: Una aproximación desde una perspectiva desarrolladora. Mendive, Revista Científico Pedagógica, 3(12), 1-6.

Caballero, M. (2007). La adaptación al Espacio Europeo de Educación Superior como escenario para la reflexión sobre el proceso de enseñanza-aprendizaje en el ámbito universitario: Algunas propuestas para un cambio significativo. Revista Española de Orientación y Psicopedagogía, 18(2), 167-177.

Callejas, M., \& Vitalia, M. (2002). La renovación de los estilos pedagógicos: Colectivos para la investigación y la acción en la universidad. Revista de Docencia Universitaria, 3(1), 1-22. 
Camargo, A., \& Hederich, C. (2007). Los estilos de enseñanza. Un concepto en búsqueda de precisión. Pedagogía y Saberes, 26, 3140.

Clariana, M. (2001). Las preferencias instruccionales: Conceptualización y evaluación. Revista de Psicología General y Aplicada, 54(2), 259-277.

Coll, C., \& Onrubia, J. (1993). El análisis del discurso y la construcción de significados compartidos en aula. Revista Latina de Pensamiento y Lenguaje, 1(2), 241-259.

Compagnucci, E., Cardós, P., \& Ojeda, G. (2002). Acerca de las prácticas docentes y la enseñanza de la psicología. Revista de Teoría y Didáctica de las Ciencias Sociales, 7, 7-24.

De la Fuente Arias, J., Salvador Granados, M., \& De la Fuente Arias, M.(1992). Conocimientos psicológicos, competencia conductual y estilo docente. Revista Interuniversitaria de Formación del Profesorado, 15, 145158.

De León, I. (2005). Los estilos de enseñanza pedagógicos: Una propuesta de criterios para su determinación. Revista de Investigación, 57, 69-97.

Delgado, M. A. (1991). Los estilos de enseñanza en la educación física. Propuesta para una reforma de la enseñanza. Granada, España: I.C.E. Universidad de Granada.

Duit, R. (2006). La investigación sobre la enseñanza de las ciencias. Un requisito imprescindible para mejorar la práctica educativa. Revista Mexicana de Investigación Educativa, 11(30), 741770.

Durán, E., \& Costaguta, R. (2008). Experiencia de enseñanza adaptada al estilo de aprendizaje de los estudiantes en un curso de simulación. Formación Universitaria, 1(1), 19-28.

Feixas, M. (2010). Enfoques y concepciones docentes en la universidad. RELIEVE, 16(2), 1-27.

Felder, R., \& Silverman, L. (1988). Learning and teaching styles in engineering education. Engineering Education, 78(7), 674-681.

Figueroa, N., \& Vigliecca, M. (2006). Reflexiones sobre nuevos enfoques de enseñanza en ingeniería a partir de las experiencias con estilos de aprendizaje. Revista de Informática Educativa y Medios Audiovisuales, 3(7), 32-36.

Finson, K., Thomas, J., \& Pedersen, J. (2006). Comparing science teaching styles to students' perceptions of scientists. School Science \& Mathematics, 106(1), 8-15.

Gagliardi, R. (2008). Gestión de la educación técnica-profesional: Capacitación directiva para la formación de jóvenes autónomos. Buenos Aires, Argentina: Noveduc.

García García, J., \& Perales Palacios, F. (2005). ¿Afectan los usos didáctico y científico de las gráficas cartesianas a su comprensión? Un estudio con alumnos de bachillerato y universidad. Didáctica de las ciencias experimentales y sociales, $19,57-74$

Gargallo, B. (2008). Estilos de docencia y evaluación de los profesores universitarios y su influencia sobre los modos de aprender de sus estudiantes. Revista Española de Pedagogía, 241, 425446.

Gené, A. (1998). ¿Educar en la universidad? En J. Porta \& M. Llandosa (Coords.), La Universidad en el cambio de siglo (pp. 121-138). Madrid, España: Alianza.

Guzmán, J. (2009). ¿Cómo enseñan psicología los profesores efectivos? Un estudio exploratorio. Perfiles Educativos, 30(123), 8-26.

Guzmán, J. (2011). La calidad de la enseñanza en 
educación superior. ¿Qué es una buena enseñanza en este nivel educativo? Perfiles Educativos, 33(Núm. Esp), 129141.

Hervás, M. (2008). Identificación de variables que influyen en los estilos de aprendizaje. Claves para conocer cómo aprenden los estudiantes. Revista Estilos de Aprendizaje, 1, 143-167.

Irigoyen, J., Jiménez, M., \& Acuña, K. (2003). Nuevas tecnologías y educación. Enseñanza e Investigación en Psicología, 8(2), 203-216.

Irigoyen, J., Jiménez, M., \& Acuña, K. (2004). Evaluación del ejercicio instruccional en la enseñanza universitaria. Enseñanza e Investigación en Psicología, 9(2), 293302.

Laudadio, J. (2012). Evaluación de estilos de enseñanza en la universidad: Estudio preliminar de las propiedades psicométricas del Cuestionario sobre la Orientación Docente del Profesor Universitario (CODPU). Interdisciplinaria, 29(1), 79-93.

Martínez, P. (2009). Estilos de enseñanza: Conceptualización e investigación. Revista Estilos de Aprendizaje, 3, 3-19.

Mosquera, J., \& Furió-Más, C. (2008). El cambio didáctico en profesores universitarios de química a través de un programa de actividades basado en la enseñanza por investigación orientada. Didáctica de las ciencias experimentales y sociales, 22, 115-154.

Muros Molina, J., Som Castillo, A., Leyva Rodríguez, A., \& Zabala Díaz, M. (2010). Efecto de dos estilos de enseñanza (cognoscitivo versus tradicional) sobre el aprendizaje de conceptos de anatomía muscular en alumnos de Educación Física de $1^{\text {o }}$ de ESO. Apunts: Educación física y deportes, $100,23-31$.
Ortiz, E., \& Mariño, M. (2007). La psicodidáctica como enfoque interdisciplinario del proceso de enseñanza-aprendizaje universitario. Revista Pedagogía Universitaria, 12(3), 32-45.

Pastor Martínez, M. (2010). Estilos de aprendizaje y estilos de enseñanza: De alumna a maestra. Encuentro: Revista de investigación e innovación en la clase de idiomas, 19, 96-102.

Petrucci, D., \& Dibar, M. (2001). Imagen de la ciencia en alumnos universitarios: Una revisión y resultados. Enseñanza de las Ciencias, 19(2), 217-229.

Pibernat Riera, L. (2010). Fundamentación epistémica del acto de enseñanza. Bases para la didáctica de la historia y la geografía. Enseñanza de las ciencias sociales, 9, 115-128.

Pinelo, F. (2008). Estilos de enseñanza de los profesores de la carrera de Psicología. Revista Mexicana de Orientación Educativa, 5(13), 17-24.

Polanco, R. (1999). El estilo de aprendizaje como predictor del desempeño docente en profesores de áreas administrativosociales y de ingeniería. Revista Latinoamericana de Psicología, 31(3), 527-536.

Pozo, J., Sanz, A., Gómez, M., \& Limón, M. (1991). Las ideas de los alumnos sobre la ciencia: Una interpretación desde la psicología cognitiva. Enseñanza de las Ciencias, 9(1), 83-94.

Pulido Martos, M., De la Torre-Cruz, M., Luque Ramos, P., \& Palomo Monereo, A. (2009). Estilos de enseñanza y aprendizaje en el EEES: un enfoque cualitativo. Revista Estilos de Aprendizaje, 4, 156-171.

Ramírez Díaz, M., \& Chávez Lima, E. (2010). Análisis de la influencia del estilo de enseñanza del profesor en el aprendizaje de estudiantes de física 
a nivel universitario. Latin-American Journal of Physics Education, 4(Supl.1), 1002-1008.

Ramsden, P. (1993). Learning to teach in higher education. London: Routledge.

Rendón, M. (2010a). Los estilos de enseñanza en la Universidad de Antioquia (Primera etapa Facultad de Educación). UniPluri/versidad, 10(1), 1-18.

Rendón, M. (2010b). Una descripción de los estilos de enseñanza en la Universidad de Antioquia. Uni-Pluri/versidad, 10(2), 1-19.

Rosales, A. (2004). Estrategias didácticas o de intervención docente en el área de la educación física. Lecturas: Educación física y deportes, 75, 1-5.

Roselli, N. (2011). Variabilidad intraindividual e (in)dependencia epistémica del docente en el desarrollo de clases expositivas y participativas-guiadas. Revista Investigaciones en Psicología -UNMSM, 14(1), 31-52.

Salvador, L., Argos González, J., Ezquerra Muñoz, M., Osoro Sierra, M., \& Castro, A. (2011). Perfiles de estilos de aprendizaje de los estudiantes universitarios y metodologías docentes. Bordón. Revista de pedagogía, 63(2), 41-52.

Suárez, C., \& Elias, C. (Coord.). (2008). Los estilos pedagógicos y su impacto en el aprendizaje de los alumnos (20012008). Bogotá: Fondo de Publicaciones Universidad Sergio Arboleda.

Tárraga Mínguez, R., Martínez Monfort, P., Gómez del Amo, R., Pastor Cerezuela, G., \& Fernández Andrés, I. (2012). Análisis de las preferencias instruccionales de estudiantes universitarios. El papel del material de apoyo a las clases. Revista Iberoamericana de Informática Educativa, 16, 69-77.

Valadez, M. (2009). Estilos de aprendizaje y estilos de pensamiento: Precisiones conceptuales. Revista Educación y Desarrollo, 11, 19-30.

Ventura, A.C. (2011). Estilos de aprendizaje y prácticas de enseñanza en la universidad. Un binomio que sustenta la calidad educativa. Perfiles Educativos, 33(Esp.), 142-154.

Viale, H. (2007). Menos es más: Cómo propiciar el aprendizaje autónomo mediante una clase integral en el marco del modelo pedagógico UPC. Revista Digital de Investigación en Docencia Universitaria, 3(1), 1-22.

Viale, H. (2011). Organización de la clase: ¿Preparo mi clase para enseñar o para que el alumno aprenda? Revista Digital de Investigación en Docencia Universitaria, 5(1), 1-32. 